\title{
Review
}

\section{Potential serological biomarkers of cerebral malaria}

\author{
Naomi W. Lucchi ${ }^{\mathrm{a}, \mathrm{b}}$, Vidhan Jain ${ }^{\mathrm{c}}$, Nana O. Wilson ${ }^{\mathrm{d}}$, Neeru Singh ${ }^{\mathrm{c}}$, \\ Venkatachalam Udhayakumar ${ }^{\mathrm{a}, \mathrm{b}}$ and Jonathan K. Stiles ${ }^{\mathrm{d}, *}$ \\ ${ }^{a}$ Atlanta Research and Education Foundation, Decatur, GA, USA \\ ${ }^{\mathrm{b}}$ Malaria Branch, Division of Parasitic Diseases and Malaria, Center for Global Health, Centers for Disease \\ Control and Prevention, Atlanta, GA, USA \\ ${ }^{\mathrm{c}}$ National Institute of Malaria Research, Regional Medical Research Center for Tribals, Jabalpur, India \\ ${ }^{\mathrm{d}}$ Morehouse School of Medicine, Atlanta, GA, USA
}

\begin{abstract}
Biomarkers have been used to diagnose and prognosticate the progress and outcome of many chronic diseases such as neoplastic and non communicable diseases. However, only recently did the field of malaria research move in the direction of actively identifying biomarkers that can accurately discriminate the severe forms of malaria. Malaria continues to be a deadly disease, killing close to a million people (mostly children) every year. One life-threatening complication of malaria is cerebral malaria (CM). Studies carried out in Africa have demonstrated that even with the best treatment, as high as 15-30\% of CM patients die and about $10-24 \%$ of CM survivors suffer short-or long-term neurological impairment. The transition from mild malaria to $\mathrm{CM}$ can be sudden and requires immediate intervention. Currently, there is no biological test available to confirm the diagnosis of CM and its complications. It is hoped that development of biomarkers to identify CM patients and potential risk for adverse outcomes would greatly enhance better intervention and clinical management to improve the outcomes. We review here what is currently known regarding biomarkers for CM outcomes.

A Pub Med literature search was performed using the following search terms: "malaria," "cerebral malaria," "biomarkers," "mortality" and "neurological sequelae." This search revealed a paucity of usable biomarkers for CM management. We propose three main areas in which researchers can attempt to identify CM biomarkers: 1) early biomarkers, 2) diagnostic biomarkers and 3) prognostic biomarkers.
\end{abstract}

Keywords: Biomarkers, cerebral malaria, severe malaria, malaria

\section{Biomarkers in disease management}

A biomarker is a substance or a characteristic that can be objectively measured and evaluated as an indicator of normal biologic processes, pathogenic processes or responses to a therapeutic intervention. Biomarkers have been used to diagnose and prognosticate the progress and outcome of many diseases. They can be

${ }^{*}$ Corresponding author: Jonathan K. Stiles, Morehouse School of Medicine, Department of Microbiology, Biochemistry and Immunology, BMSB Room 349D, 720 Westview Dr. SW, Atlanta, GA 30310, USA. Tel.: +1 404742 1586; Fax: +1 404752 1179; E-mail: jstiles @msm.edu. used to support disease management and control in many different aspects: 1) to diagnose a disease, 2) to identify "at risk" individuals, 3 ) to stratify patients depending on disease severity, 4 ) to provide prognosis of the disease, 5) to assess disease severity, 6) to provide some guidance in the treatment and management of a disease and eventually 7 ) to identify at risk patients for long term complication after the manifestation of a particular illness. Biomarkers can be found in any biological fluids such as serum, plasma, urine, cells or they can be biological products such as metabolites, cytokines or genetic markers. An example of an ideal biomarker is the measurement of hemoglobin as an indicator of anemia. This is a simple, quantitative and 
inexpensive procedure, therefore allowing for wide usage. Biomarkers have become valuable for the diagnosis and prognosis of many chronic diseases such as cancer, diabetes, autoimmune diseases and HIV/AIDS. However, the use of biomarkers in parasitic infectious diseases is limited.

The use of biomarkers in clinical practices requires a thorough validation of their utility. Pepe et al. proposed at least five phases of biomarker development for cancer [1] and recently this was adapted for acute kidney injury by Coca and Parikh [2]. Briefly, the initial phase involves preclinical exploration during which potential biomarkers are identified. Markers capable of discriminating the severity of the disease are identified at this phase. The second phase involves the development of clinical assay (s) capable of measuring the potential biomarkers; these assays are established to detect the disease and validate the biomarkers. In the third phase, retrospective longitudinal studies are carried out to test the new biomarkers for their utility. Possibly the biomarkers should detect the disease early enough before it becomes clinically obvious and during this phase, the biomarkers' sensitivity and specificity are determined. The fourth phase involves a prospective screening phase which entails the use of the identified biomarkers to screen large populations to determine their utility. Finally, the fifth phase involves determination of the biomarker's impact on disease control and management with a successful biomarker having a tangible impact on the management of the disease.

\section{Malaria manifestations}

Approximately 2 billion people are exposed to malaria with morbidity surpassing 250 million cases and close to 800,000 deaths per year [3]. Depending on the transmission intensities and the age of the patient, malaria manifests with different clinical outcomes ranging from asymptomatic malaria, mild uncomplicated disease to life threatening severe disease. In malaria endemic areas such as Africa, where transmission is high, studies have revealed a large number of Plasmodium falciparum-infected individuals showing no clinical signs of malaria [4-7]. The prevalence of these asymptomatic malaria cases has been shown to be as high as $52 \%$ using molecular diagnostic tools (such as PCR) for diagnosis [7]. Symptomatic malaria in adults in these endemic regions is mainly characterized by febrile illness with no complications (mild malaria (MM)). Severe malaria illness such as cerebral malar- ia (CM), severe anemia (SA) and respiratory distress syndrome are also observed, especially in children less than five years of age. In contrast, in regions where malaria transmission is low and/or seasonal, such as some parts of Asia and South America, all age groups are at risk for malaria and its severe forms. Severe malaria (SM) often manifests with a different disease spectrum according to local epidemiology and malaria prevalence. For example, in low malaria transmission areas of Asia and South America CM, severe malaria anemia (SMA), acute renal failure (also known as acute kidney injury), jaundice and multi organ failure are common while in Africa, severe manifestations include CM, SMA, respiratory distress and acidosis [8]. While it was previously thought that only P. falciparum caused SM, recent reports have challenged this notion by demonstrating that severe and complicated malaria is also observed with $P$. vivax infection [9-17] underscoring the huge public health impact of SM.

Biomarkers have been used to diagnose and prognosticate the progress and outcome of many chronic and acute infectious, metabolic and non communicable diseases. The transition from MM to the severe forms of malaria can be sudden and requires immediate intervention. Therefore, the use of biomarkers to risk-stratify severe malaria patients would greatly enhance patient care and assist in appropriate management of health care resources. In addition, biomarkers that identify asymptomatic cases, who might have levels of parasitemia undetectable by light microscopy or other conventional testing methods, will be crucial for monitoring the elimination of malaria reservoirs from endemic populations [18]. Therefore, biomarkers for malaria management are clearly a necessary tool for malaria control programs.

\section{Why biomarkers for cerebral malaria?}

Cerebral malaria is one of the life-threatening complications of malaria and is defined as an unarousable coma with a $P$. falciparum infection in the absence of other causes of encephalopathy. If left untreated, CM is fatal in 24-72 hours. The incidence of CM is estimated to be $1,120 / 100,000$ per year in endemic malaria areas of Africa where children below the age of five bear the biggest burden (reviewed in [19]). About 30\% of CM patients die even after treatment with effective antimalarial therapy, while up to $24 \%$ of the survivors develop neurological complications and cognitive impairment post recovery [20-23] (and reviewed in [24]). 


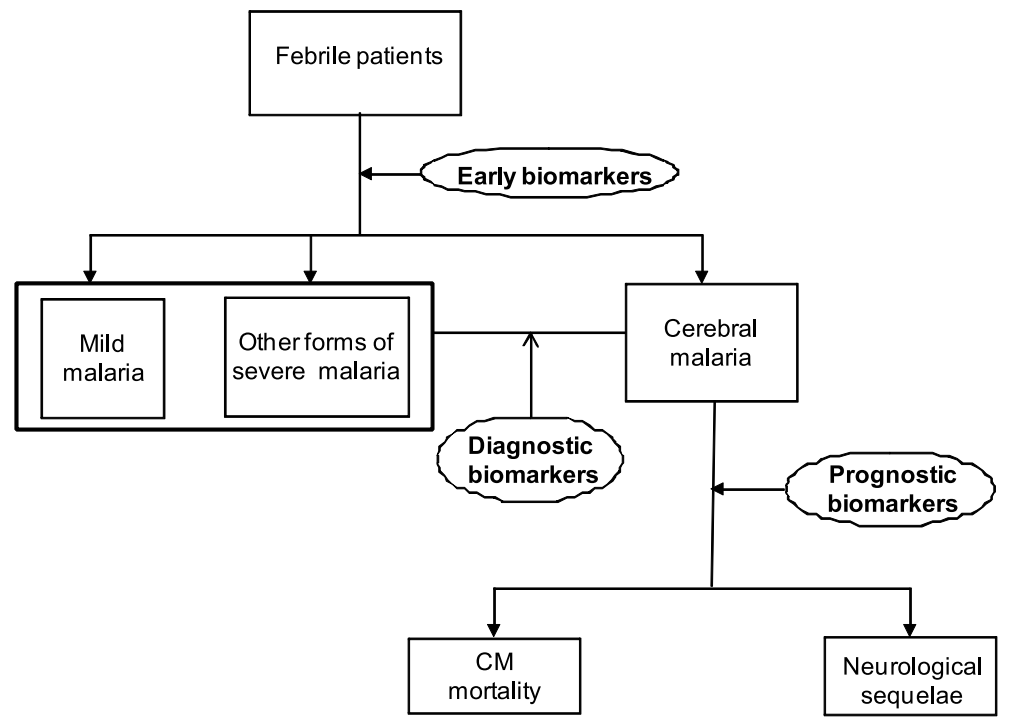

Fig. 1. Proposed use of biomarkers for cerebral malaria management. Three categories of biomarkers would be necessary for the management of CM: 1) early biomarkers capable of identifying febrile patients at risk for CM on admission, 2) diagnostic biomarkers that can unequivocally identify $\mathrm{CM}$ patients and 3) prognostic biomarkers that can identify $\mathrm{CM}$ patients at risk for death and those at risk for neurological impairments post recovery.

CM patients require immediate intensive care and intervention with parenteral quinine or, as recently demonstrated, artesunate [25]. Therefore, early diagnosis and subsequent referral to tertiary heath care facilities are crucial for patient survival. An accurate diagnosis of $\mathrm{CM}$ is equally crucial, especially since a few studies have suggested that in some cases malaria parasitemia is incidental to other severe infections such as pneumonia. Indeed, post mortem results have demonstrated that up to $23 \%$ of children previously diagnosed as CM cases died of other causes [26]. Identification of usable biomarkers for the reliable identification of CM would provide a "tool" that would be useful for the early detection, diagnosis and prognosis of CM. This would eventually assist in provision of appropriate patient care to improve clinical outcome.

While no clear biomarker exists for CM management, to date, recent studies have identified serological factors that have the potential of being biomarkers. More studies are needed to validate the utility of these biomarkers. We review here what we believe to be potential biomarkers for CM management and hypothesize on their use.

\section{Proposed biomarkers for cerebral malaria}

Phase 1 of biomarker identification starts with some understanding of the pathogenesis of the disease and an exploratory phase in which different factors that can clearly discriminate different levels of disease severity are identified. The pathogenesis of CM is still not well understood although it is clearly multifactorial, involving sequestration of $P$. falciparum infected erythrocytes to brain vasculature, triggering inflammatory cytokine responses and apoptotic pathway leading to a breach and dysfunction of the blood brain barrier, tissue damage and repair [26-29]. In an attempt to understand the pathogenesis of CM, our previous studies in Ghana [30] and India [31] for the first time revealed a striking association between the chemokine interferon inducible protein-10 (CXCL10-) and CM severity, suggesting that CXCL10 may be a biomarker for CM severity. We believe that the management of $\mathrm{CM}$ will require at least three main categories of biomarkers: early biomarkers, diagnostic biomarkers and prognostic biomarkers (Fig. 1).

\subsection{Early/predictive biomarkers}

Only a subset of malaria patients develops CM with the rest developing asymptomatic or mild forms of the disease, or other forms of SM such as SMA. Risk factors for $\mathrm{CM}$ include age less than 10 years and living in malaria endemic regions. However, it is not known if other unknown risk factors exist that would facilitate the identification of "at risk" patients during hospital visits before they succumb to CM. Early/predictive 
biomarkers would allow heath care workers to stratify febrile patients into those at risk for CM and those who are likely to develop MM or other forms of SM. The "at risk" patients would then be started early on the appropriate treatment and on any adjunctive measures available or referred to better health facilities as required. To date, no such biomarkers exist and clearly more studies are required in this area.

\subsection{Diagnostic biomarkers}

Diagnostic biomarkers are biomarkers that can be used to categorically identify CM patients. Currently, the diagnosis of $\mathrm{CM}$ relies on clinical indicators characterized mainly by (1) unarousable coma (no localizing response to pain) that persists for more than six hours after experiencing a generalized convulsion; (2) presence of asexual forms of P. falciparum; and (3) exclusion of other causes of encephalopathy (e.g. viral, bacterial). Other clinical algorithms and indicators have been investigated. One of these is malaria retinopathy, whose usefulness has been tested in different parts of Africa [32-35] and India [36,37]. Many of these studies have shown malaria retinopathy to be a good diagnostic indicator capable of distinguishing CM patients from patients with other causes of coma in parasitemic comatose patients (reviewed in [38]).

In addition, several serological factors have been identified that are differentially expressed in CM patients compared to either MM patients or healthy controls. Our group and others have demonstrated that distinct profiles of cytokine and/or chemokine are associated with discrete clinical manifestation of falciparum malaria [30,31,39,40]. For example, increased levels of CXCL10, sFas, sTNF-R2 [30,31], IL-8, IL-1ra [39], and decreased levels of RANTES [40] and vascular endothelial growth factor (VEGF) [31] were all found in CM patients and not in MM or health control cases. The endothelial regulators, angiopoietin-1 (ANG1) and ANG-2 were also shown to be differentially expressed in CM compared to MM patients [41,42]. Some of these discriminatory factors have also been observed in cerebral spinal fluid (CSF) samples [30]. Our studies in Ghana demonstrated that higher levels of CSF CXCL10 and lower levels of VEGF were found in CM patients compared to MM or healthy controls [30].

Many of the factors discussed above can be viewed as being in the phase 1 of biomarker development during which preclinical exploration of potential biomarkers is performed and markers capable of discriminating the severity of the disease are identified. Perhaps the only serological factors analyzed for their predictive value for CM diagnosis, to date, are the endothelial regulators, ANG-1 and ANG-2 [41,42] and the chemokine CXCL10 [43]. Angiopoietins are known to regulate the maintenance of vascular integrity and were found to be differentially regulated in two different populations of CM patients from Thailand and Africa [42]. The levels of ANG-1 were significantly reduced in CM patients compared to healthy controls and MM patients and, conversely, ANG-2 levels were significantly elevated in $\mathrm{CM}$ patients compared to control groups [42]. Receiver Operating Curve (ROC) analyses were performed to test the sensitivity and specificity of these factors, as discriminatory biomarkers for the accurate diagnosis of CM. ANG-1, ANG-2 and the ratio of ANG-2/ANG-1 were all shown to accurately discriminate between $\mathrm{CM}$ and MM patients [42]. Indeed, ANG-1 levels were independently associated with CM even in a multivariate logistic regression model. In the same study, TNF levels were shown to be significantly increased in $\mathrm{CM}$ compared to MM patients in the Thai adult population, but this factor did not discriminate well in the Uganda children [42]. This finding demonstrates the importance of testing the utility of any potential biomarker using different populations and different age groups as the sensitivity and specificity might be affected by the transmission intensity and host immunity.

\subsection{Prognostic biomarkers}

More often than not, appropriate treatment of CM leads to complete patient recovery. However, an unacceptably high number (up to $30 \%$ ) of CM patients die regardless of receiving the recommended treatment and up to $24 \%$ of CM survivors develop neurological complications and cognition problems post-recovery [2023]. Prognostic biomarkers to predict CM outcomes may be life-saving as the "at risk" patients can be given the necessary interventions and/or adjunctive therapies to prevent the adverse CM outcomes. These biomarkers can be divided into two main groups: those that can predict neurological sequelae post recovery and those that predict $\mathrm{CM}$ mortality. To date, some potential biomarkers that can be used to predict $\mathrm{CM}$ adverse outcomes have been suggested although their utility needs to be validated.

\subsection{Biomarkers to predict risk of developing neurological sequelae post-recovery}

Identifying persons at risk for neurological sequelae will assist in providing the necessary remedy at dis- 
charge or as early as is necessary and possible. For example, a recent study demonstrated that computerized cognitive training for children who survived CM provided some benefit on some neuropsychological and behavioral functions even long after the malaria episode [44]. Therefore, this remedy, or others, can be administered to those patients at risk for the development of neurological deficit post-recovery. Neuroprotective factors such as erythropoietin (EPO) are critical in the brain repair process $[45,46]$ and are therefore important for recovery from any brain insult. A recent study using African children demonstrated that high plasma EPO levels were associated with a $70 \%$ reduction in the risk of being discharged with neurological sequelae [47]. EPO levels above 200 units/liter were independently associated with about an $80 \%$ reduction in risk of developing neurological sequelae in a matched analysis [47]. The beneficial effects of EPO occur locally in the tissue or the brain via EPO receptors found in neurons, astrocytes, microglia and endothelial cells [48], and previous studies have shown that EPO can actively translocate across the blood brain barrier [49]. From the point of view of biomarker development, serological EPO levels would provide a usable biomarker for development of neurological sequelae with CM patients having $<200 \mathrm{U} /$ liter (or another cutoff found to be more appropriate) of plasma EPO being at risk. Validation of this hypothesis is required using large studies in different endemic regions and using different age groups.

\subsection{Biomarkers to predict fatal CM}

While a biomarker to predict fatal CM would serve for prognosis, it would be of more value if interventions or adjunctive therapies are available to save the patients. Using these biomarkers, clinicians can provide the additional intervention before it is too late. Inflammatory cytokines induce brain injury and cause apoptotic death of brain tissue components and several studies have shown that some of these are associated solely with CM mortality. In a study conducted in India, we evaluated the role of the ratio of some apoptotic and angiogenic factors in discriminating between the different malaria disease groups. Increased levels of CXCL10, sTNF-R2 and sFas were associated with disease severity being highest in the CM non-survivors [31]. The role of CXCL10 in CM mortality was further confirmed using a murine model of CM [50] in which, CXCL10 and CXCL9 (both CXCR3 ligands) were shown to be highly induced in the brains of mice infected with $P$. berghei
ANKA. Mice deficient in these ligands were protected from experimental CM-related death [50] and accordingly, CXCL10 and CXCL9 knockout mice were shown to be partially protected from CM associated death [50]. Elevated plasma levels of CXCL10 and CXCL4 were tightly associated with CM mortality, and ROC analysis revealed that these chemokines can discriminate CM-non survivors from MM $(p<0.0001)$ and CM-survivors $(p<0.0001)$ with an area under the curve $($ AUC) $=1$ [43]. In addition, other studies revealed that CXCL10 independently predicted severe and fatal CM [30,31,50,51] as elevated levels of CXCL10 expression in the CSF and peripheral blood plasma were observed in $\mathrm{CM}$ patients who died compared to $\mathrm{CM}$ survivors.

Other factors shown to influence CM outcome include circulating levels of VEGF, a factor known to protect neuronal compartments from injury and death [30, 31], ANG-1 and ANG-2/ANG-1 ratio. CM patients who died had the lowest level of VEGF compared to CM-survivors [31]. The study by Jain et al. further demonstrated that the mean and median ratios of CXCL10/VEGF, sTNFR2/VEGF, and sFas/VEGF increased as the disease severity increased, with the highest ratio occurring in the CM-non survivor group. ANG-1 levels at presentation were also associated with higher risk of mortality in African children, while ANG-2/ANG-1 ratios were higher in those patients who subsequently died of CM [42]. All these studies point to the fact that these serological factors can be used to predict fatal CM.

\section{Discussion}

As summarized in Table 1, recent studies have demonstrated that the development of CM-management biomarkers is possible. If we were to adapt Pepe's biomarker development stages, the development of the different categories of the CM-management biomarker can be viewed as being at different phases. Clearly, the development of early biomarkers is in the initial stages and a lot of effort will be needed to identify biomarkers that can distinguish febrile patients likely to develop CM. One wonders if some of the elevated immune factors reported to be found in CM patients and not in MM patients can be detected before the development of CM. If this is the case, then potentially these could be tested for their utility as early biomarkers. Both the diagnostic and prognostic biomarkers are in the second and third phases of biomarker development and the va- 
Table 1

Summary of available potential CM biomarkers

\begin{tabular}{ll}
\hline Type of biomarker & Potential Biomarkers \\
\hline $\begin{array}{l}\text { Early biomarkers that can predict febrile patients who will succumb } \\
\text { to CM }\end{array}$ & None \\
$\begin{array}{ll}\text { Diagnostic biomarker that can identify CM patients } & \text { ANG-2/ANG-1 ratio, decreased ANG-1 levels increased ANG-2 } \\
& \text { levels [42] } \\
& - \text { Increased levels of CXCL10 [43]. } \\
\text { Prognostic biomarkers for development of neurological sequelae } & - \text { EPO levels }>\text { 200 U per liter [47] } \\
& \\
\begin{array}{l}\text { Prognostic biomarker that can identify patients at risk for CM- } \\
\text { associated mortality }\end{array} & - \text { Increase CXCL10/VEGF, sTNFR2/VEGF, sFas/VEGF [31] } \\
& - \text { Increased ANG-2 levels [42,61] } \\
& - \text { increased levels of CXCL4 and CXCL10 [43] }\end{array}$ \\
\hline
\end{tabular}

lidity of all these potential biomarkers will need to be explored using large prospective multicenter studies to clearly define their utility (phase 4). Eventually, the role of these biomarkers in CM management will have to be determined (phase 5), during which time questions such as "is the use of biomarkers helping to improve the clinical management of CM or treatment outcomes (reducing mortality outcomes)?" will be answered.

Importantly, the usage of biomarkers will be highly relevant if remedies for severe $\mathrm{CM}$ outcomes (or severe malaria for that matter) are available. Therefore, investing in biomarker development may also contribute to better understanding of the biological process involved in the pathogenesis and this could eventually lead to development of novel adjunctive therapies for CM management. For example, angiogenic factors have long been implicated as prognostic markers in cerebral ischemia [52] and, additionally, these same factors have been found to be therapeutic. Indeed, the role of EPO as an adjunctive treatment for CM patients has been proposed [53]. EPO administration was shown to increase the survival of mice during murine experimental CM [54] and the administration of EPO in humans has been tried with no adverse effects reported [55]. In our studies, both in India and Ghana, we observed that CM patients who died had low VEGF levels [30,31], implying that VEGF may be required for tissue recovery. If these findings are confirmed in future studies then one could consider any molecule that can mimic the function of VEGF as a potential candidate for adjunctive therapy. Further studies on the role of these factors for adjunctive therapies are required.

Single biomarkers are rarely used except in some exceptional cases such as the marker for anemiahemoglobin level. Most likely for CM, as in many diseases, a composite biomarker panel will be necessary. For example, the panel for the prognostic biomarkers could consist of CXCL10, CXCL4, ANG-1, ANG-2,
EPO, VEGF, sTNFR2 and sFas. The development of biomarkers is a long journey that will require some technological tools along the way. Once validated, the format required for the identified biomarkers will become an issue. Ideally, the panel of biomarkers should be detected in a rapid diagnostic format allowing for a quick and easy-to-use tool in malaria endemic regions. Probably an appropriate format would be a microchip whereby detection of the biomarkers is done on a chip whose results are detected using a simple, point-of-care (bedside) device or field deployable device. Serum obtained from CM patients can be tested for the presence of the biomarkers and from the results of either absolute levels and/or ratios of some of the factors, health care workers or clinicians will be able to risk stratify the patients. Accordingly, the patients can be given any available intervention to meet their needs such as the computerized cognitive training for children, demonstrated to provide some benefit on some neuropsychological and behavioral functions after a CM episode [44].

The use of biomarkers for the management of SM is being recognized not just for patients in endemic areas, but also in travelers such as military personnel, expatriates, tourists and the like who present with imported malaria upon returning to their countries. This is especially crucial since many clinicians in these countries are not accustomed to diagnosing malaria. The utility of plasma lactate levels [56] and that of both neopterin and procalcitonin [57] as biomarkers for SM at the initial clinical assessment of travelers with imported malaria was tested in recent studies. In the latter study, however, the diagnostic value of neopterin and procalcitonin was limited and the authors proposed the use of these factors for exclusion of SM in order to assist clinicians' decisions on subsequent treatment or referral to specialized facilities [57].

One of the challenges for advocating the utility of biomarkers in the clinical management of malaria is 
the cost involved. As most of the severe disease occurs in resource limited endemic countries, there is always concern as to whether expensive detection methods can be used as practical tools. While this is a reasonable concern, the recent development of simpler and more field appropriate detection tools [58,59] indicates that reliable biomarkers can also be measured using appropriate simple methods. It is hoped that such appropriate technology will be adapted for biomarker detection when these are available.

In this review, we have highlighted what is currently known about biomarkers for CM management and hypothesized on their use. However, biomarkers can be useful in the early diagnosis of other severe forms of malaria (e.g., SMA or severe malaria in general) as recently proposed by Erdman et al. [60]. In addition, biomarkers could aid in the detection of asymptomatic cases which, if left untreated, act as reservoirs for malaria parasites. However, to date, no such markers exist. In summary, the use of biomarkers as an additional "tool" for malaria management would clearly enhance clinical management of severe malaria patients and improve treatment outcomes. It is hoped that this review has demonstrated the significance of developing novel biomarkers as new tools for the management of $\mathrm{CM}$ and highlighted new developments that are emerging in this field to realize such a goal.

\section{Acknowledgement}

This investigation received financial support from WHO/UNDP/TDR Collaborative Research Grant (A00 524) and National Institutes of Health grant numbers NIH-FIC (R21TW006804-01), NIH-RCMI (RR03034) and NIH-NIGM-MBRS (SO6GM08248). NWL is supported by Atlanta Research and Education Foundation.

\section{References}

[1] M.S. Pepe, R. Etzioni, Z. Feng, J.D. Potter, M.L. Thompson et al., Phases of biomarker development for early detection of cancer, J Natl Cancer Inst 93 (2001), 1054-1061.

[2] S.G. Coca and C.R. Parikh, Urinary biomarkers for acute kidney injury: perspectives on translation, Clin J Am Soc Nephrol 3 (2008), 481-490.

[3] World Malaria Report, 2008. World Health Organization.

[4] F.N. Baliraine, Y.A. Afrane, D.A. Amenya, M. Bonizzoni, D.M. Menge et al., High prevalence of asymptomatic plasmodium falciparum infections in a highland area of western Kenya: a cohort study, J Infect Dis 200 (2009), 66-74.
[5] S. Mabunda, J.J. Aponte, A. Tiago and P. Alonso, A countrywide malaria survey in Mozambique. II. Malaria attributable proportion of fever and establishment of malaria case definition in children across different epidemiological settings, Malar J 8 (2009), 74.

[6] M. Vafa, M. Troye-Blomberg, J. Anchang, A. Garcia and F. Migot-Nabias, Multiplicity of Plasmodium falciparum infection in asymptomatic children in Senegal: relation to transmission, age and erythrocyte variants, Malar J 7 (2008), 17.

[7] M.P. Dal-Bianco, K.B. Koster, U.D. Kombila, J.F. Kun, M.P. Grobusch et al., High prevalence of asymptomatic Plasmodium falciparum infection in Gabonese adults, Am J Trop Med Hyg 77 (2007), 939-942.

[8] W.P. O’Meara, P. Bejon, T.W. Mwangi, E.A. Okiro, N. Peshu et al., Effect of a fall in malaria transmission on morbidity and mortality in Kilifi, Kenya, Lancet 372 (2008), 1555-1562.

[9] M.J. Barcus, H. Basri, H. Picarima, C. Manyakori, Sekartuti et al., Demographic risk factors for severe and fatal vivax and falciparum malaria among hospital admissions in northeastern Indonesian Papua, Am J Trop Med Hyg 77 (2007), 984-991.

[10] B. Genton, V. D’Acremont, L. Rare, K. Baea, J.C. Reeder et al., Plasmodium vivax and mixed infections are associated with severe malaria in children: a prospective cohort study from Papua New Guinea, PLoS Med 5 (2008), e127.

[11] D.K. Kochar, A. Das, S.K. Kochar, V. Saxena, P. Sirohi et al., Severe Plasmodium vivax malaria: a report on serial cases from Bikaner in northwestern India, Am J Trop Med Hyg 80 (2009), 194-198.

[12] D.K. Kochar, S.K. Kochar, R.P. Agrawal, M. Sabir, K.C. Nayak et al., The changing spectrum of severe falciparum malaria: a clinical study from Bikaner (northwest India), $J$ Vector Borne Dis 43 (2006), 104-108.

[13] D.K. Kochar, D. Pakalapati, S.K. Kochar, P. Sirohi, M.P. Khatri et al., An unexpected cause of fever and seizures, Lancet 370 (2007), 908

[14] D.K. Kochar, V. Saxena, N. Singh, S.K. Kochar, S.V. Kumar et al., Plasmodium vivax malaria, Emerg Infect Dis 11 (2005), 132-134.

[15] M.K. Mohapatra, K.N. Padhiary, D.P. Mishra and G. Sethy, Atypical manifestations of Plasmodium vivax malaria, Indian J Malariol 39 (2002), 18-25.

[16] J. Prakash, A.K. Singh, N.S. Kumar and R.K. Saxena, Acute renal failure in Plasmodium vivax malaria, J Assoc Physicians India 51 (2003), 265-267.

[17] E. Tjitra, N.M. Anstey, P. Sugiarto, N. Warikar, E. Kenangalem et al., Multidrug-resistant Plasmodium vivax associated with severe and fatal malaria: a prospective study in Papua, Indonesia, PLoS Med 5 (2008), e128.

[18] B. Ogutu, A.B. Tiono, M. Makanga, Z. Premji, A.D. Gbadoe et al., Treatment of asymptomatic carriers with artemetherlumefantrine: an opportunity to reduce the burden of malaria? Malar J 9, 30.

[19] R. Idro, K. Marsh, C.C. John and C.R. Newton, Cerebral malaria: mechanisms of brain injury and strategies for improved neurocognitive outcome, Pediatr Res 68 (2008), 267274.

[20] M.J. Boivin, P. Bangirana, J. Byarugaba, R.O. Opoka, R. Idro et al., Cognitive impairment after cerebral malaria in children: a prospective study, Pediatrics 119 (2007), e360-366.

[21] J.A. Carter, J.A. Lees, J.K. Gona, G. Murira, K. Rimba et al., Severe falciparum malaria and acquired childhood language disorder, Dev Med Child Neurol 48 (2006), 51-57.

[22] J.A. Carter, A.J. Ross, B.G. Neville, E. Obiero, K. Katana et 
al., Developmental impairments following severe falciparum malaria in children, Trop Med Int Health 10 (2005), 3-10.

[23] R. Idro, J.A. Carter, G. Fegan, B.G. Neville and C.R. Newton, Risk factors for persisting neurological and cognitive impairments following cerebral malaria, Arch Dis Child 91 (2006), $142-148$.

[24] V. Mung'Ala-Odera, R.W. Snow and C.R. Newton, The burden of the neurocognitive impairment associated with Plasmodium falciparum malaria in sub-saharan Africa, Am J Trop Med Hyg 71 (2004), 64-70.

[25] A.M. Dondorp, C.I. Fanello, I.C. Hendriksen, E. Gomes, A. Seni et al., Artesunate versus quinine in the treatment of severe falciparum malaria in African children (AQUAMAT): an openlabel, randomised trial, Lancet 376, 1647-1657.

[26] T.E. Taylor, W.J. Fu, R.A. Carr, R.O. Whitten, J.S. Mueller et al., Differentiating the pathologies of cerebral malaria by postmortem parasite counts, Nat Med 10 (2004), 143-145.

[27] H. Armah, A.K. Dodoo, E.K. Wiredu, J.K. Stiles, A.A. Adjei et al., High-level cerebellar expression of cytokines and adhesion molecules in fatal, paediatric, cerebral malaria, Ann Trop Med Parasitol 99 (2005), 629-647.

[28] I.A. Clark, K.A. Rockett and W.B. Cowden, Role of TNF in cerebral malaria, Lancet 337 (1991), 302-303.

[29] B.Y. Sarfo, S. Singh, J.W. Lillard, Jr., A. Quarshie, R.K. Gyasi et al., The cerebral-malaria-associated expression of RANTES, CCR3 and CCR5 in post-mortem tissue samples, Ann Trop Med Parasitol 98 (2004), 297-303.

[30] H.B. Armah, N.O. Wilson, B.Y. Sarfo, M.D. Powell, V.C. Bond et al., Cerebrospinal fluid and serum biomarkers of cerebral malaria mortality in Ghanaian children, Malar J 6 (2007), 147.

[31] V. Jain, H.B. Armah, J.E. Tongren, R.M. Ned, N.O. Wilson et al., Plasma IP-10, apoptotic and angiogenic factors associated with fatal cerebral malaria in India, Malar J 7 (2008), 83.

[32] N.A. Beare, C. Southern, C. Chalira, T.E. Taylor, M.E. Molyneux et al., Prognostic significance and course of retinopathy in children with severe malaria, Arch Ophthalmol 122 (2004), 1141-1147.

[33] C. Hirneiss, V. Klauss, M. Wilke, A. Kampik, T. Taylor et al., [Ocular changes in tropical malaria with cerebral involvementresults from the Blantyre Malaria Project], Klin Monbl Augenheilkd 222 (2005), 704-708.

[34] S. Lewallen, H. Bakker, T.E. Taylor, B.A. Wills, P. Courtright et al., Retinal findings predictive of outcome in cerebral malaria, Trans R Soc Trop Med Hyg 90 (1996), 144-146.

[35] S. Lewallen, T.E. Taylor, M.E. Molyneux, B.A. Wills and P. Courtright, Ocular fundus findings in Malawian children with cerebral malaria, Ophthalmology 100 (1993), 857-861.

[36] D.K. Kochar, Shubhakaran, B.L. Kumawat, I. Thanvi, A. Joshi et al., Ophthalmoscopic abnormalities in adults with falciparum malaria, QJM 91 (1998), 845-852.

[37] D.K. Kochar, Shubhakaran, B.L. Kumawat and S.P. Vyas, Prognostic significance of eye changes in cerebral malaria, $J$ Assoc Physicians India 48 (2000), 473-477.

[38] N.A. Beare, T.E. Taylor, S.P. Harding, S. Lewallen and M.E. Molyneux, Malarial retinopathy: a newly established diagnostic sign in severe malaria, Am J Trop Med Hyg 75 (2006), 790-797.

[39] C.C. John, A. Panoskaltsis-Mortari, R.O. Opoka, G.S. Park, P.J. Orchard et al., Cerebrospinal fluid cytokine levels and cognitive impairment in cerebral malaria, Am J Trop Med Hyg 78 (2008), 198-205.

[40] C.C. John, R. Opika-Opoka, J. Byarugaba, R. Idro and M.J. Boivin, Low levels of RANTES are associated with mortality in children with cerebral malaria, J Infect Dis 194 (2006), 837-845.

[41] A.L. Conroy, E.I. Lafferty, F.E. Lovegrove, S. Krudsood, N. Tangpukdee et al., Whole blood angiopoietin-1 and -2 levels discriminate cerebral and severe (non-cerebral) malaria from uncomplicated malaria, Malar J 8 (2009), 295.

[42] F.E. Lovegrove, N. Tangpukdee, R.O. Opoka, E.I. Lafferty, N. Rajwans et al., Serum angiopoietin-1 and -2 levels discriminate cerebral malaria from uncomplicated malaria and predict clinical outcome in African children, PLoS One 4 (2009), e4912.

[43] N.O. Wilson, V. Jain, C.E. Roberts, N. Lucchi, P.K. Joel et al., CXCL4 and CXCL10 predict risk of fatal cerebral malaria, Dis Markers 30, 39-49.

[44] P. Bangirana, B. Giordani, C.C. John, C. Page, R.O. Opoka et al., Immediate neuropsychological and behavioral benefits of computerized cognitive rehabilitation in Ugandan pediatric cerebral malaria survivors, J Dev Behav Pediatr 30 (2009), 310-318.

[45] A.L. Siren and H. Ehrenreich, Erythropoietin - a novel concept for neuroprotection, Eur Arch Psychiatry Clin Neurosci 251 (2001), 179-184.

[46] A.L. Siren, M. Fratelli, M. Brines, C. Goemans, S. Casagrande et al., Erythropoietin prevents neuronal apoptosis after cerebral ischemia and metabolic stress, Proc Natl Acad Sci U S A 98 (2001), 4044-4049.

[47] C. Casals-Pascual, R. Idro, N. Gicheru, S. Gwer, B. Kitsao et al., High levels of erythropoietin are associated with protection against neurological sequelae in African children with cerebral malaria, Proc Natl Acad Sci U S A 105 (2008), 2634-2639.

[48] M. Bernaudin, A. Bellail, H.H. Marti, A. Yvon, D. Vivien et al., Neurons and astrocytes express EPO mRNA: oxygensensing mechanisms that involve the redox-state of the brain, Glia 30 (2000), 271-278.

[49] M.L. Brines, P. Ghezzi, S. Keenan, D. Agnello, N.C. de Lanerolle et al., Erythropoietin crosses the blood-brain barrier to protect against experimental brain injury, Proc Natl Acad Sci U S A 97 (2000), 10526-10531.

[50] G.S. Campanella, A.M. Tager, J.K. El Khoury, S.Y. Thomas, T.A. Abrazinski et al., Chemokine receptor CXCR3 and its ligands CXCL9 and CXCL10 are required for the development of murine cerebral malaria, Proc Natl Acad Sci U S A $\mathbf{1 0 5}$ (2008), 4814-4819.

[51] C.Q. Nie, N.J. Bernard, M.U. Norman, F.H. Amante, R.J. Lundie et al., IP-10-mediated $\mathrm{T}$ cell homing promotes cerebral inflammation over splenic immunity to malaria infection, PLoS Pathog 5 (2009), e1000369.

[52] T. Hayashi, K. Deguchi, S. Nagotani, H. Zhang, Y. Sehara et al., Cerebral ischemia and angiogenesis, Curr Neurovasc Res 3 (2006), 119-129.

[53] C. Casals-Pascual, R. Idro, S. Picot, D.J. Roberts and C.R. Newton, Can erythropoietin be used to prevent brain damage in cerebral malaria? Trends Parasitol 25 (2009), 30-36.

[54] L. Wiese, C. Hempel, M. Penkowa, N. Kirkby and J.A. Kurtzhals, Recombinant human erythropoietin increases survival and reduces neuronal apoptosis in a murine model of cerebral malaria, Malar J 7 (2008), 3.

[55] S. Picot and A.L. Bienvenu, [Plasmodium vivax infection: not so benign], Med Sci (Paris) 25 (2009), 622-626.

56] P.J. van Genderen, I.M. van der Meer, J. Consten, P.L. Petit, T. van Gool et al., Evaluation of plasma lactate as a parameter for disease severity on admission in travelers with Plasmodium falciparum malaria, J Travel Med 12 (2005), 261-264. 
[57] P.L. Witt, G.T. Spear, M.J. Lindstrom, H.A. Kessler, E.C. Borden et al., 2',5'-oligoadenylate synthetase, neopterin and beta 2-microglobulin in asymptomatic HIV-infected individuals, AIDS 5 (1991), 289-293.

[58] N.W. Lucchi, A. Demas, J. Narayanan, D. Sumari, A. Kabanywanyi et al., Real-time fluorescence loop mediated isothermal amplification for the diagnosis of malaria, PLOS ONE 5, e13733.

[59] W.G. Lee, Y.G. Kim, B.G. Chung and U. Demirci, Khademhosseini A Nano/Microfluidics for diagnosis of infectious diseases in developing countries, Adv Drug Deliv Rev 62, 449-457.

[60] L.K. Erdman, A. Dhabangi, C. Musoke, A.L. Conroy, M. Hawkes et al., Combinations of host biomarkers predict mortality among Ugandan children with severe malaria: a retrospective case-control study, PLOS ONE 6, e17440.

[61] T.W. Yeo, D.A. Lampah, R. Gitawati, E. Tjitra, E. Kenangalem et al., Angiopoietin-2 is associated with decreased endothelial nitric oxide and poor clinical outcome in severe falciparum malaria, Proc Natl Acad Sci U S A 105 (2008), 17097-17102. 


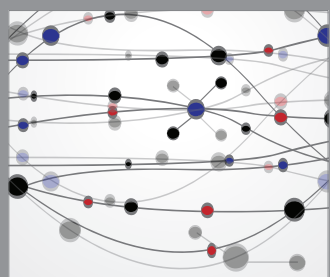

The Scientific World Journal
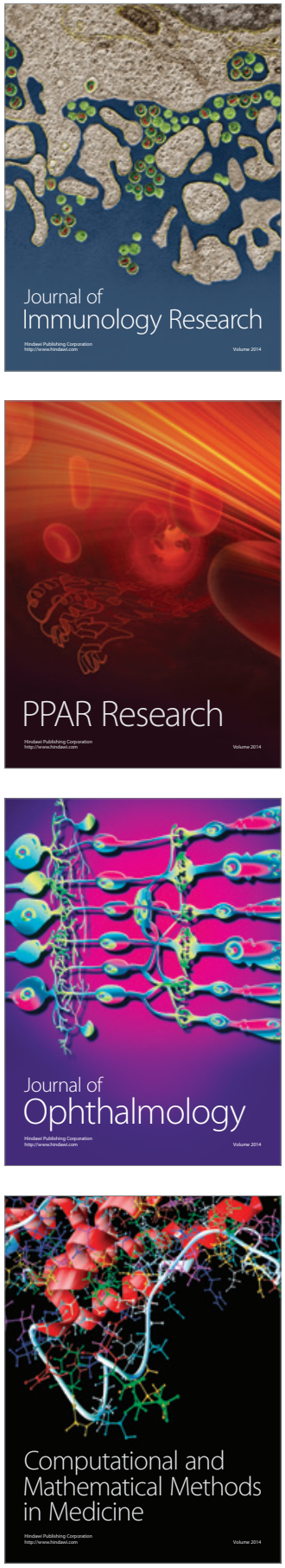

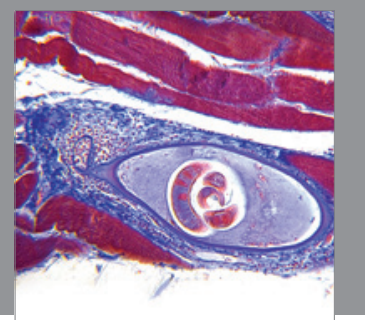

Gastroenterology

Research and Practice
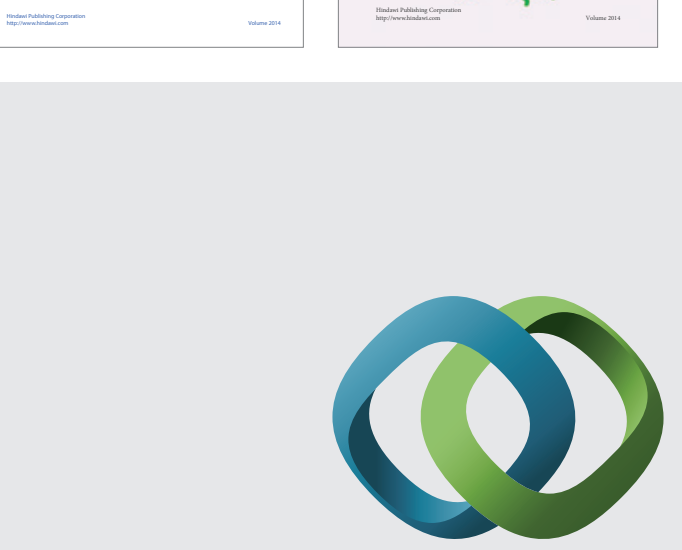

\section{Hindawi}

Submit your manuscripts at

http://www.hindawi.com
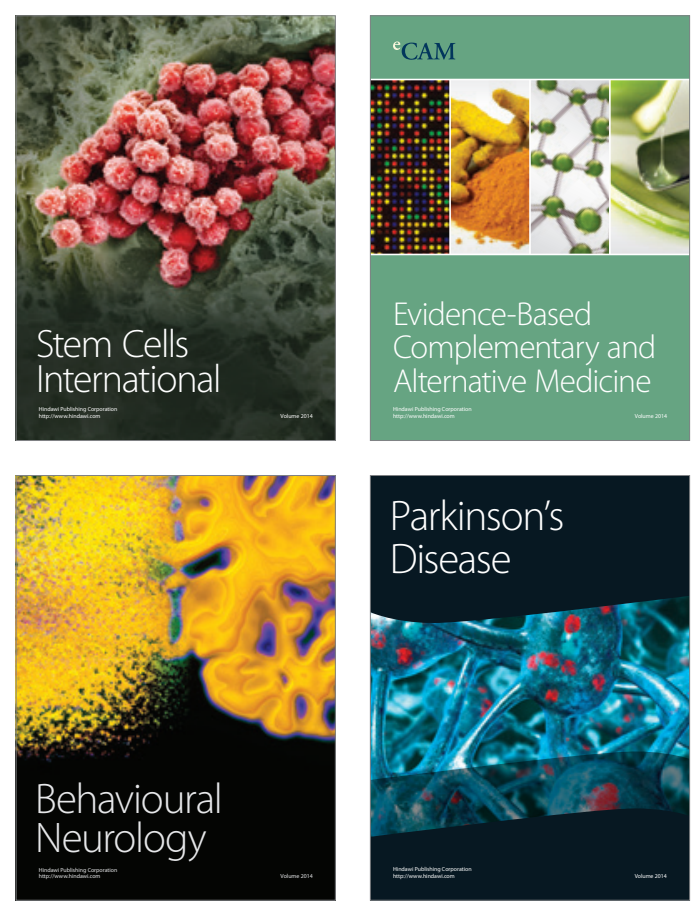

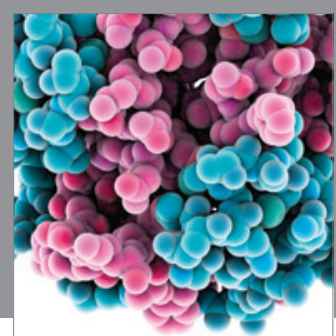

Journal of
Diabetes Research

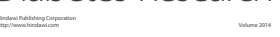

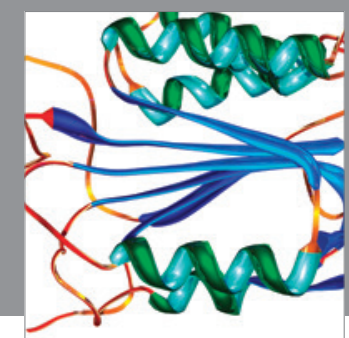

Disease Markers
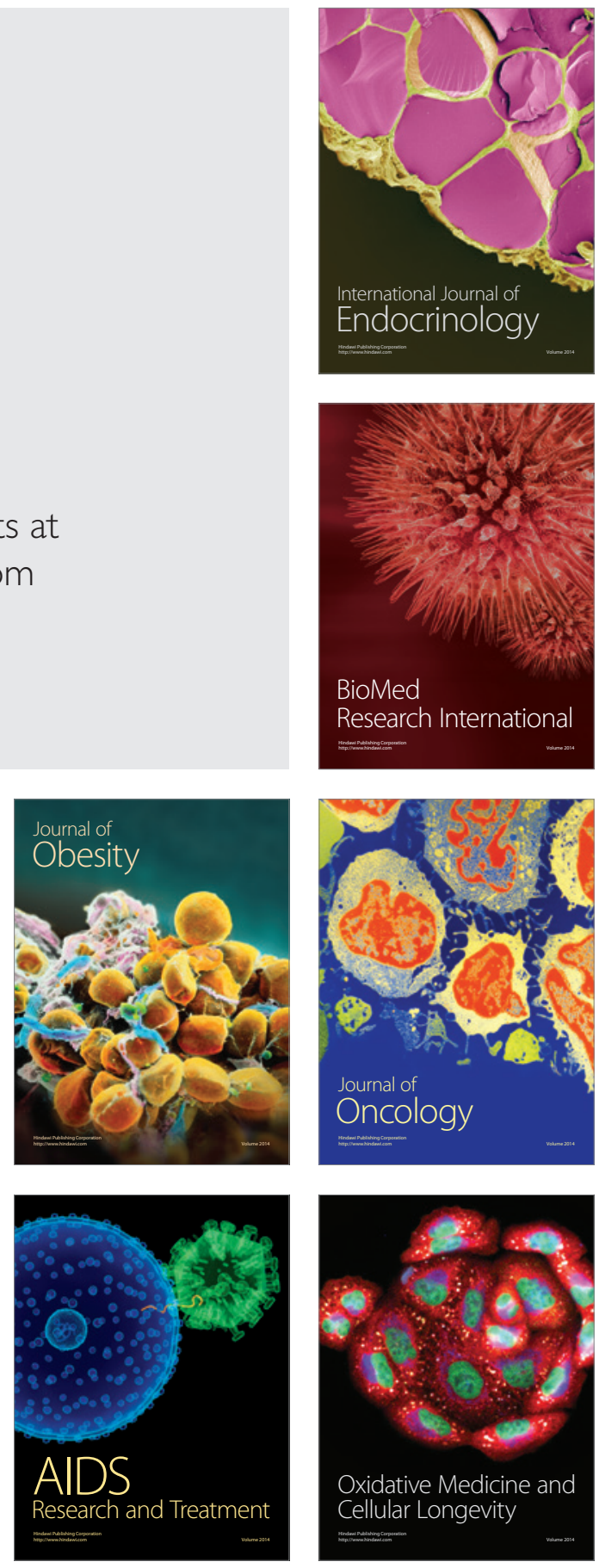